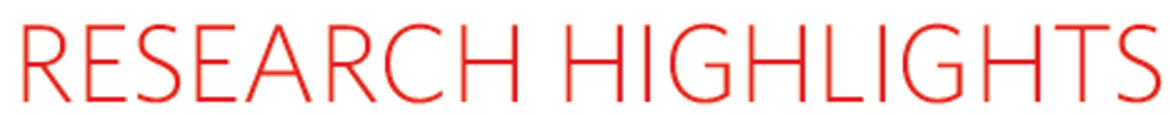

\title{
A change of weather
}

Nature Phys. doi:10.1038/nphys314 (2006)

The physics that describes the onset of tropical downpours shows intriguing similarities to theories of magnetism, physicists in the United States report.

Somematerialsbecomemagnetic only when they are cooled below a critical temperature: their magnetism arises as the spins of thematerial's electrons lineup. This change is described as a continuous phase transition.

Ole Peters of the Santa Fe Institute in New Mexico and David Neelin of the University of California, Los Angeles, used satellite data to analyse the relationship between water vapour and rainfall. The weather system undergoes a phase transition mathematically equivalent to that in magnetized materials when rising vapour levels trigger rainfall $-\mathbf{a}$ surp rising result given the difference in scale between the two systems.

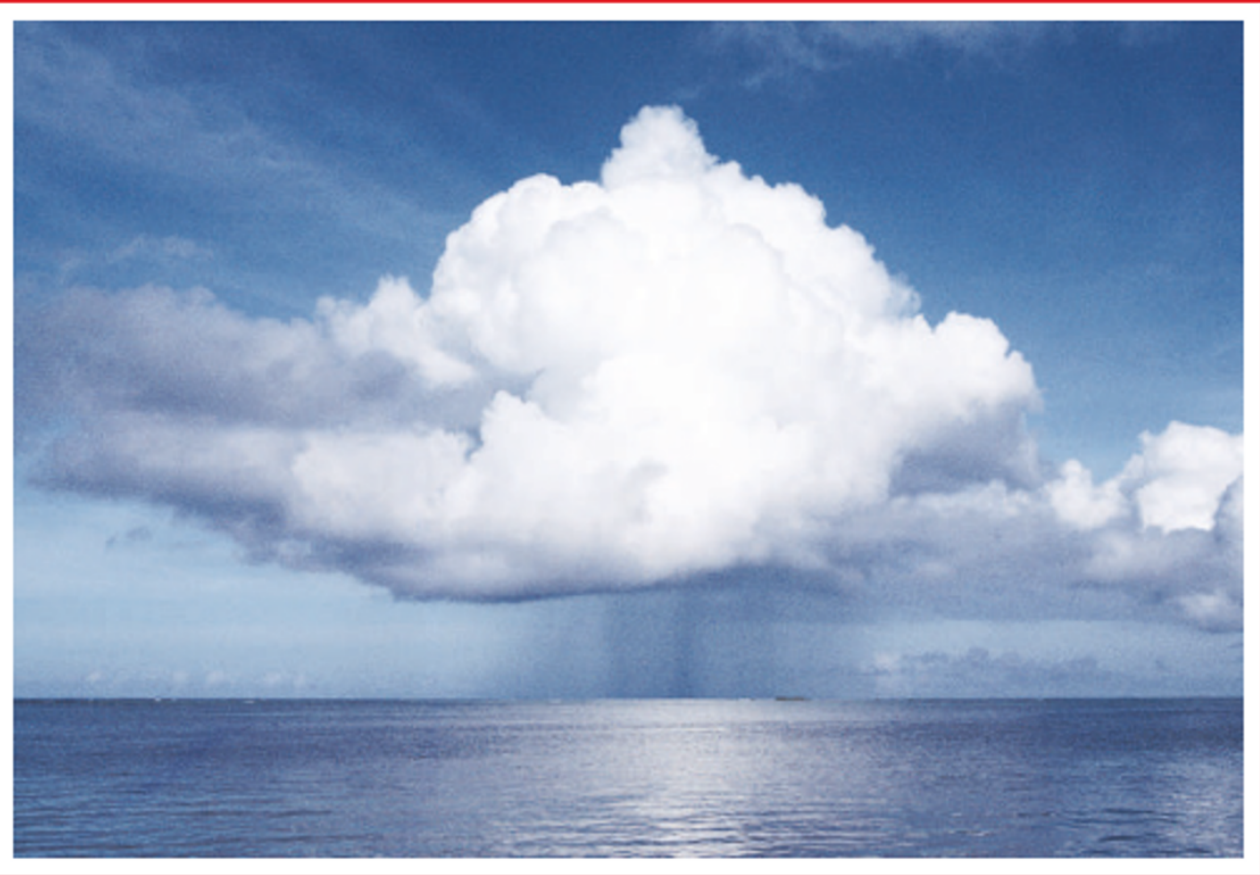

\section{CANCER BIOLOGY}

\section{The power of $\mathrm{p} 53$}

\section{Science doi:10.1126/science.1126863(2006)}

Researchers in the United States have linked the tumour-suppressor gene $p 53$ with an oddity of cancer cells that was first noted in the 1930 s, known as the Warburg effect.

Normal cells mostly make energy by aerobic respiration, using oxygen to break down sugars. The Warburg effect describes cancer cells' preference for anaerobic breakdown of sugars, a process known as glycolysis.

Paul Hwang at the National Institutes 3 of Health in Bethesda, Maryland, and his colleagues show that $p 53$ plays a part in shifting the balance by modulating a

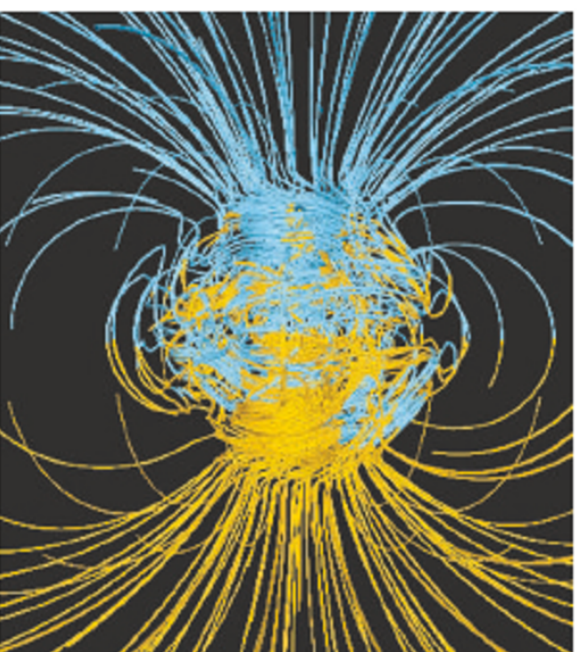

mitochondrial protein that regulates oxygen use in the cell. Mouse and human cell lines deficient in either p53 or mitochondrial protein activity were found to favour anaerobic over aerobic metabolism.

$p 53$ 's new-found link to respiration may also offer clues to the gene's role in ageing.

\section{EARTH SCIE NCE}

\section{Magnetic flip}

Earth Planet Sci. Lett. doi:10.1016/j.epst.2006.03.038 (2006)

Earth's magnetic field (pictured left) is prone to flip: north and south have swapped, on average, every half-million years for the past 160 million years. But the fact that it's been longer since the last reversal doesn't mean that one is overdue, argue Catherine Constable of the University of California, San Diego, and Monika Korte of Germany's National Research Centre for Geosciences in Potsdam.

Some researchers have speculated that the weakening of Earth's magnetic field since measurements began in the mid-nineteenth century points to an imminent reversal. Constable and Korte counter that both archaeomagnetic data and palaeomagnetic records retrieved from sediment cores show the recent rate of change to be nothing unusual. The team also notes that the current field strength is still higher than the geological average and that stronger fields have, in the past, been associated with longer reversal periods.

\section{QUANTUM COSMOLOGY}

\section{Knots in space}

Phys. Rev. Lett. 96, 180604 (2006)

The rapid expansion and cooling of the very early Universe may have created knot-like 'topological defects' in the fabric of space-time - a process that has now been mimicked in the lab using a pair of niobium rings.

When rapidly cooled, the niobium rings plunge into a superconducting state that can contain quantum-mechanical defects, analogous to cosmological defects, known as vortices. Roberto Monaco of the University of Salerno in Italy and his co-workers have examined how the probability of vortex formation depends on the cooling rate. They find that the observed relationship matches the predictions made in the 1980 s for cosmic topological defects.

\section{PHYSIOLOGY}

\section{Hormonal pain}

\section{l Neurosci. 26,5777-5785(2006)}

A brain-imaging study provides new evidence of oestrogen's role in pain perception.

Jon-Kar Zubieta of the University of Michigan in Ann Arbor and his colleagues have previously linked sex differences in pain perception to the regulation of $\mu$-opioid receptors. This receptor system, when activated by sustained pain, mediates the body's natural pain-killing mechanisms.

Now Zubieta and his team have used positron emission tomography and a label of 
$\mu$-opioid receptors to examine eight women's responses to pain in certain regions of the brain before and after treatment with oestradiol, a form of oestrogen. They conclude that oestradiol increases the number of $\mu$-opioid receptors and enhances their activation. Subjects that had been treated with oestradiol also reported less pain sensation.

\section{CHEMISTRY}

\section{Carbon united}

Angew. Chem. Int. Edn doit10.1002/anie.200600951 (2006)

Chemists have used electricity to make carbon-carbon bonds, a crucial step in building organic molecules, in a microreactor.

In doing so, Stephen Haswell of the University of Hull, UK, and his co-workers combined two beneficial strategies: electrochemistry unites carbon atoms under much milder conditions than are used in more typical metal-catalysed reactions; and microreactors offer a simple route to continuous reactions, carried out as molecules flow through tiny channels.

The researchers reacted various benzyl bromides with a series of alkenes, achieving high yields of products useful in making compounds such as antibiotics.

\section{SYSTEMS NEUROSCIE NCE}

\section{A touching story}

Neuron 50,617-629(2006)

Biologists in Switzerland have watched in real time how the behaviour of mice affects how their brains process sensory signals coming from their whiskers.

Carl Petersen and his team at the Swiss Federal Institute of Technology, Lausanne, used voltage-sensitive dyes and a lightweight fibre-optic bundle to watch patches of neurons in the part of the brain that processes whisker signals. Usually mice are anaesthetized before their neuronal activity is measured, but this technique works on mice that are awake and free to move.

The researchers showed that the neurons in still mice responded much more strongly to a whisker touch than the neurons in mice that were swishing their whiskers back and forth.

\section{MATERIA LS SCIENCE}

\section{Tight squeeze}

Science 312, 1199-1202(2006)

Appl. Phys. Lett. 88, 193121(2006)

Metals stored inside carbon nanotubes can be squeezed out like toothpaste from a tube, according to Florian Banhart of the University of Mainz, Germany, and his co-workers.
Banhart had previously shown that 'nanoonions' - multilayered concentric spheres of graphitic carbon - shrink when electron irradiation kicks out carbon atoms. Now his team reports that irradiating nanotubes filled with metal causes a toothpaste-like extrusion of their contents. The researchers estimate that the pressure created as the nanotubes contract may reach more than a tenth of that at the Earth's core.

In separate experiments, Banhart and his colleagues show how a nano-onion can be used as a high-pressure reaction cell: the internal pressure of the irradiated structure helps carbon atoms to combine with encapsulated iron, forming iron carbide.

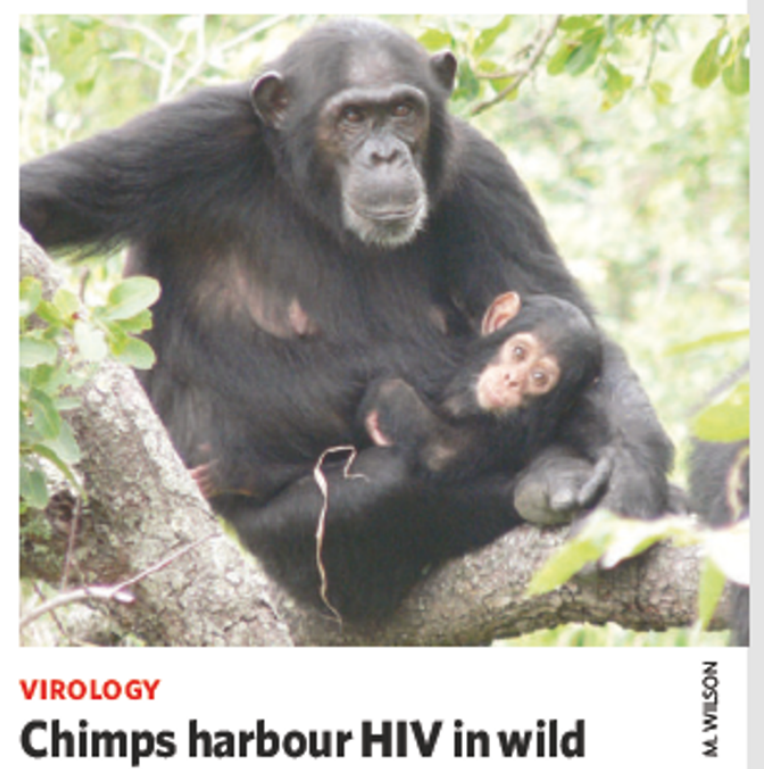

Science doi:10.1126/science.1126531 (2006)

Wild chimpanzees (pictured above) have long been suspected as the source of the human immunodeficiency virus, HIV. Now that theory has been confirmed: evidence from southern Cameroon shows that wild members of the chimpanzee subspecies Pan troglodytes troglodytes harbour the strain of simian immunodeficiency virus (SIV) that is most closely related to pandemic HIV.

Researchers led by Beatrice Hahn of the University of Alabama at Birmingham report the presence of antibodies against the SIV strain in droppings left by wild chimps. This strain had previously only been detected in captive animals.

The virus, which does not seem to cause disease in the chimps, was probably transmitted to humans by exposure to blood during bushmeat hunting or butchery. The location of the discovery also supports the idea that the HIV pandemic had its roots in nearby Kinshasa, in the Democratic Republic of Congo.

\section{JOURNAL CLUB}

\author{
Daniel Lieberman \\ Harvard University, Cambridge, \\ Massachusetts, USA
}

\section{A biological anthropologist recommends reading about optimal running, but advises against trying it.}

As someone interested in human locomotion, I frequently watch people run and walk, both on a treadmill in my lab, and on the streets of Cambridge, where l live.

It is well known that humans are good at minimizing cost: we typically walk near our optimal speed in terms of axygen cost, and switch gaits at approximately the speed for which running costs less than walking.

I often wonder how people gauge what speed to choose, and when to switch gaits. Do we base our behaviour on speed itself, the determinants of speed, such as stride length and stride frequency, or some other variables?

John Bertram of the University of Calgary in Canada and his colleagues (Gutmann et al.J. Exp. Biol 209, 622-632; 2006) recently tackled this problem - at least the running part of it - in a fun, creative experiment. They asked athletes to run at particular speeds (by using a treadmill), stride lengths (by making them land on spaced markers in a field) and stride frequencies (by making them time their footstrikes to a metronome). They then used published data to estimate the oxygen costs of the different gaits.

Interestingly, runners compensated for the constrained (and sometimes odd) gaits by adjusting the unconstrained parameters in ways that apparently minimized their oxygen intake. So even when forced to take mincing little steps, or to galumph slowly, the athletes somehow optimally adjusted their speed, stride length and/or stride rate.

How bodies figure out this trick remains a mystery. All I can say is that I've tried constraining my gait in similar ways when I run in the morning, but instead of feeling optimal, I mostly feel foolish. 